\title{
Design of a Microbiota Sampling Capsule using 3D-Printed Bistable Mechanism*
}

\author{
Mouna Ben Salem ${ }^{1,2}$, Guillaume Aiche ${ }^{1}$, Lennart Rubbert ${ }^{2}$, Pierre Renaud ${ }^{2}$ and Yassine Haddab $^{1}$
}

\begin{abstract}
Microbiota analysis is a fundamental element for a better understanding of microbiota role, its relationship with the human body and its impact on different pathologies. There is today no non-invasive tool for easy collection of the microbiota in the small intestine. In this paper, we describe the development of such a device that opens the way to new diagnostic techniques. The device is based on a capsule designed as a passive system to maximize the safety during its use. Originality of the design relies in the use of a bistable mechanism obtained using additive manufacturing in order to provide a compact design with integration of opening, sampling and closing functions within the capsule. Design, implementation and initial lab evaluation of sampling are presented.
\end{abstract}

\section{INTRODUCTION}

The gastrointestinal (GI) tract is a major organ system in the human body which hosts the microbiota. It is well established that this microbiota has an intimate relationship with the body, and that a microbial imbalance can lead to several diseases such as obesity, cancer and inflammatory bowel disease [1], [?]. The exact correlation between dysbiosis and pathologies is today to be investigated, and sampling of this substance would therefore be beneficial for both fundamental clinical research and diagnostic. The microbiota needed for diagnosis is the bacterial flora located in the small intestine. As the access to the lower part of the GI tract is limited, the conventional techniques used for sampling are invasive, uncomfortable for the patient and most of the time do not reflect the exact intestinal environment [2]. Hence the development of minimally invasive and representative microbiota sampling tool is greatly needed.

Several capsules have been developed for other medical tasks in the intestine [3]. Most of them are used for endoscopy, and some to perform actions within the body. This means they integrate active components as camera, batteries and actuators. In this case, integration of components is difficult given strong size constraints. It was yet still possible to achieve different functions, as it is interestingly presented for instance in [4], [5] with integration of drug delivery and biopsy functions. Passive sampling capsule concepts for microbiota sampling within the small intestine have been proposed in the patents [8], [7]. As an extension, we propose to develop a capsule taking advantage of a bistable

*Research supported by the Investissements d'Avenir (Labex CAMI ANR-11-LABX-0004).

M. Ben Salem, G. Aiche and Y. Haddab are with the Laboratory of Informatics, Robotics and Microelectronics, University of Montpellier, France (phone: +33 3881191 57; e-mail: bensalem, aiche, haddab@lirmm.fr)

L. Rubbert and P. Renaud are with the Engineering Science, Computer Science and Imaging Laboratory, University of Strasbourg, France (e-mail: lennart.rubbert, pierre.renaud@insa-strasbourg.fr) mechanism. Designing such sampling capsule as a passive device is of great benefit as safety is an obvious concern for such an ingestible device. In other words, the GI tract will not be exposed to any potentially harmful chemical or electrical component. Such design approach is also in favor of compactness, as we propose to store energy mechanically, without the need to integrate batteries.

Choosing a passive approach for sampling is not necessarily sufficient to solve integration constraints. In addition, we propose to design the capsule using additive manufacturing in order to ease the integration of components required for the sampling and also potentially to minimize costs with a design compatible with single use.

The paper is organized as follows. In section II] the specifications to fulfill are analyzed and then the proposed operating principle is presented. The proposed design is then introduced in section III], starting from the general principle to its implementation using 3D printing and use of PVA foam as a trigger mechanism. Initial experiments are presented in section IV before concluding on the interest of the device and further developments in section $\mathrm{V}$.

\section{SPECIFICATIONS AND CAPSULE OPERATING MODE}

\section{A. Identification of specifications}

As other capsules, the sampling capsule will be first ingested (Figure 17. This is the first phase during the use of the capsule. After that, it will follow the natural path created by the esophagus and the stomach to reach the small intestine. This is the second main phase of use. As a third phase, sampling is then performed in the small intestine followed by evacuation. Requirements are linked to each of the three phases as described below.

Ingestion of the capsule requires this latter to respect a maximum size defined as the 000 size of pharmaceutical capsules. This means the maximum diameter of the capsule is equal to $9.9 \mathrm{~mm}$, and its maximum length is equal to $26.1 \mathrm{~mm}$.

During its transit in the stomach, the integrity of the capsule must be preserved, while the stomach is an acid environment.

Once located in the small intestine, the capsule must be capable of collecting a sample. The objective is to develop a passive device. As a consequence, a requirement is that the capsule must be specifically sensitive to the $\mathrm{pH}$ of the small intestine. Another requirement is the absence of sample contamination once it entered the capsule. Closing of the capsule must avoid any fluid leakage until the device is being naturally evacuated. 


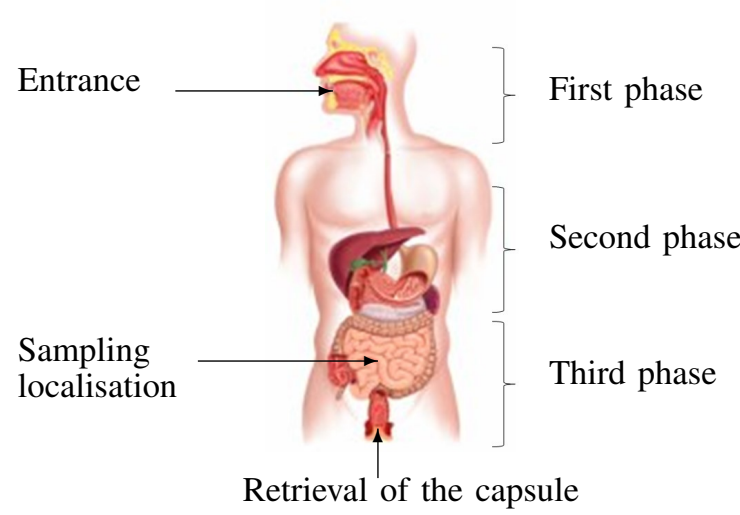

Fig. 1: The three main phases of use of the sampling capsule. Figure adapted from [6].

\section{B. Proposition of operating mode}

As the purpose of the proposed capsule is to sample microbiota, a significant volume needs to be dedicated to sample storing. We therefore choose to have the capsule passively displaced thanks to the peristaltic contractions of the GI tract, and the components of the capsule are thus only dedicated to sampling. The sequence we propose is illustrated in Figure 2 and it can be decomposed as follows.

1. The capsule is initially included into a enteric capsule, such as a gelatin pill coated with a gastro-resistant layer. It can therefore remain sealed until it reaches the small intestine (Fig. 2a).

2. When the capsule arrives in the small intestine, the $\mathrm{pH}$ of the environment increases to a more neutral $\mathrm{pH}$. The gelatin-based coating then dissolves. The thickness of the coating can be adapted to modify the time of dissolution and therefore the location of sampling in the small intestine (Fig. 2b).

3. Once the coating is completely dissolved, an opening on the outer surface of the capsule main body makes possible the intestinal liquid to enter. Sample collection is therefore achieved through this inlet orifice (Fig. 2c).

4. A porous foam is present in the capsule for microbiota sampling. This approach is based on the principle described in the patent [8], [7]. This foam forms a kind of sponge that is therefore exposed to the intestinal liquid now entering in the capsule. The sponge absorbs it and swells. We propose in addition to the sampling to use its expansion to create a force that acts on a bistable mechanism embedded in the capsule body. This mechanism acts like a trigger, that is then switched thanks to the sponge to close the inlet orifice (Fig. 2d). The interest of the bistable mechanism is twofold: first, energy is stored in the mechanism by its preload, without loss of space in the capsule. Second, the stable position corresponds to the closing of the capsule, to ensure the absence of fluid leakage.

5. Once the capsule is sealed, it can lead its way out of the human body (Fig. 2e).

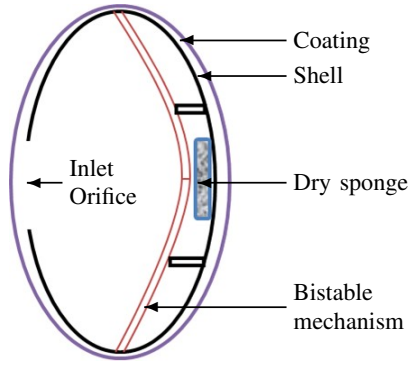

(a) Initial state of the capsule as ingested

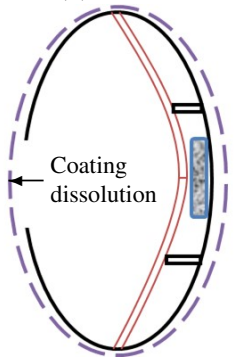

(b) Dissolution of the coating in the small intestine

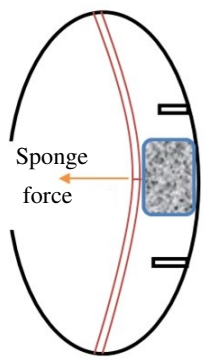

(d) Swelling of the sponge to trigger the bistable mechanism

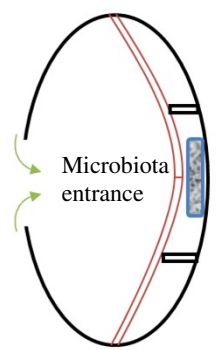

(c) Complete dissolution of the coating followed by the entry of microbiota

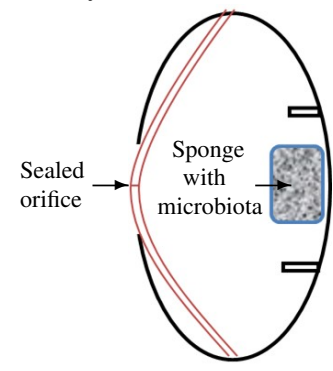

(e) Sealed capsule ready with the bistable mechanism in its first stable position
Fig. 2: Decomposition of the sampling capsule operating principle.

\section{Design AND MANufacturing}

\section{A. Key components of the capsule}

As outlined hereabove, the capsule is composed of three key components (Figure 2a): First, a shell that constitutes the main body of the capsule. It is a closed envelope, except the inlet orifice for fluid entrance. Second, a sponge that is therefore used as an actuator and a sampler at the same time. Polyvinyl Alcohol (PVA) is a highly absorbent material and expandable when wet. It is therefore suitable for absorbing intestinal fluid [7]. Third, the bistable mechanism to close the capsule.

Using 3D printing, it is possible to consider the design of the bistable mechanism and the capsule outer shell as a single component, which is interesting to respect the maximum admissible size for ingestion. This supposes however the ability to ensure the presence of bistability effect in presence of manufacturing errors, which constitues a first design issue. A second issue is the triggering of the bistable mechanism by the sponge. This supposes a correct selection of sponge and 
bistable mechanism dimensions. This is performed in two steps, with first the characterization of the sponge which acts as an actuator and second the determination of the mechanism dimensions.

\section{B. Characterization of the actuator}

Characterization of the actuator consists in determining the relationship between the sponge displacement during swelling and the force it can generate. A dedicated experimental setup was then built as illustrated in Figure 3, which is composed of a laser-based displacement sensor (Keyence LK-H152) and a flexure beam whose mechanical properties are known in order to convert the measured displacement to force evaluations.

Before testing, the sponge is compressed and placed under the flexure beam. When hydrated it swells and pushes the beam to produce a displacement that is recorded and then converted to a force. With sponges of prismatic shape, of intial dimensions equal to $10 \times 5 \times 10 \mathrm{~mm}$ and a compressed height of $2 \mathrm{~mm}$, generated forces are between $0.5 \mathrm{~N}$ and $1 \mathrm{~N}$.

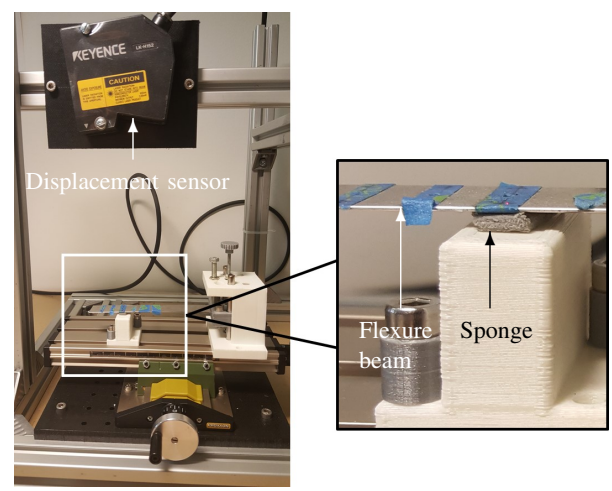

Fig. 3: Experimental setup for actuator characterization.

\section{Design of the bistable mechanism}

Design of bistable mechanisms was considered in Qiu et $a l$. [9]. We use here several results of that paper, considering an initially curved beam. In our context, such shape is indeed more interesting than a straight shape as its corresponds to a straight beam buckled in its first mode, without the need for axial preload. It is needed to constrain the second buckling mode so we consider in the end two curved beams clamped together as shown in Figure 4a

The geometry of the bistable mechanism is defined by thickness $t$, depth $b$, span $L$, initial apex height $h$ as represented in Figure $4 \mathrm{a}$. These parameters are constrained by 1 . the capsule space requirement, 2 . the force needed to close the capsule, 3. the existence of the bistable effect and 4. the triggering of the mechanism by the sponge force.

Following [9], the bistability condition is expressed by

$$
Q=\frac{h}{t} \geq 6,
$$

the force delivered by the sponge is linked to the mechanism dimensions

$$
f_{\text {top }} \approx 1480 \frac{E I h}{L^{3}}, \quad f_{\text {bot }} \approx 740 \frac{E I h}{L^{3}}
$$

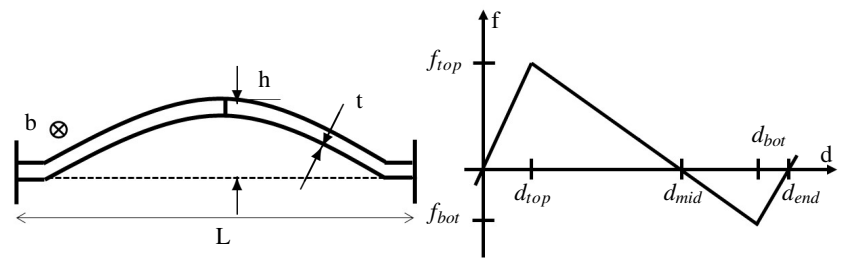

(a) Geometry

(b) Force-displacement characteristic

Fig. 4: Schematic presentation of the bistable mechanism and the evolution of the force according to the displacement [9]

with $I$ the second moment of area, $E$ the Young's modulus, and the force-displacement relationship is described as represented in Figure $4 \mathrm{~b}$ with

$d_{\text {top }} \approx 0.16 h, \quad d_{\text {bot }} \approx 1.92 h, \quad d_{\text {end }} \approx 1.99 h, \quad d_{\text {mid }} \approx 1.33 h$

From these relationships and the conditions expressed hereabove, the geometric parameters are determined and reported in Table $\mathrm{I}$. The corresponding CAD design is represented in Figure 5 .

TABLE I: Bistable mechanism dimensions after synthesis

\begin{tabular}{|c||c|c|c|c|}
\hline Parameter & t & b & L & h \\
\hline Value $(\mathrm{mm})$ & 0.35 & 2 & 18 & 2.4 \\
\hline
\end{tabular}

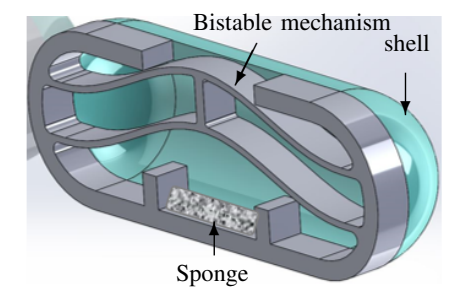

Fig. 5: CAD presentation of the capsule. Sponge is represented before swelling, and the bistable mechanism before the preload.

\section{Manufacturing}

Previous synthesis of the bistable mechanism was performed with assumption of linear and isotropic behavior of the material. Since 3D printed materials can exhibit anisotropy, an experimental evaluation of the manufacturing impact was performed. Fused Deposition Modeling technique (FDM, Fortus $400 \mathrm{MC}$, Stratasys Ltd) is being used for manufacturing, as it allows the production of biocompatible materials. Polycarbonate (PC) was used as material for the initial prototype to validate the concept. As for the final prototype it will be manufactured using ABS M30i (acrylonitrile butadiene styrene) which is biocompatible and sterilizable. Manufacturing of prototypes of the bistable mechanism showed first that the junction between the two curved beams is a weak point in the structure. Second, the ends of the curved beams present flexibilities that impact the bistable 
behavior. Slight modifications of the intersection between the two beams and their ends was consequently achieved. Initial manufacturing of the capsule showed that the accuracy could also impact the assembly of the capsule with a clearance between the two half shells of the capsule. Additional lugs were then integrated to guarantee the precision during the assembly. Example of the capsule components after these modifications are shown in Figure 6

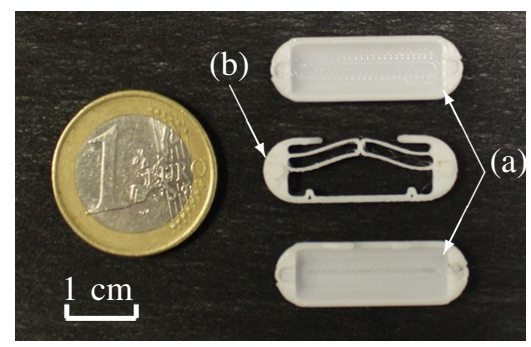

Fig. 6: Printed components of the microbiota sampling capsule, (a): shell, (b): bistable mechanism

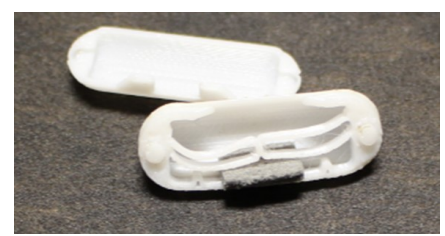

(a) Capsule assembly and integration of the dry sponge

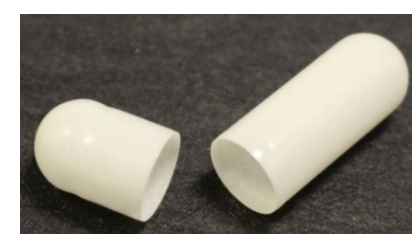

(b) Gelatin-based coating for the sampling capsule
Fig. 7: Close-ups on the capsule main components before lab evaluation

\section{EXPERIMENTAL EVALUATION}

After assembly, the bistable mechanism simply needs to be preloaded in the second stable position while the sponge is compressed and added under the mechanism (Fig. 7a). The capsule can then be closed and inserted in the gelatin-based capsule (Fig. 7b).

Since the capsule is dedicated to sampling, the experimental evaluation consisted in immersion of the capsule in a beaker filled with water. Figures $8 \mathrm{a}$ and $8 \mathrm{~b}$ show the first phase, with the air exiting the capsule through the inlet orifice to allow the entry of liquid in the capsule. This liquid entry is continuous and completed in $4 \mathrm{~min} 40 \mathrm{sec}$. Figure $8 \mathrm{c}$ correspond to the end of the filling process. Fourteen seconds later, the orifice is closed by the bistable mechanism as shown in Figure $8 \mathrm{~d}$.

After the experiment, the capsule was being inspected. We could observe the swelling of the sponge, that reached its maximum possible volume as visible in Fig. 9. A separate experiment showed the sponge is capable of absorbing $200 \mu L$, which represents approximately $22.210^{6}$ of sampled bacteria considered to be sufficient for microbiota analysis.

\section{Conclusion}

In this paper, a capsule for microbiota sampling was proposed. The capsule is passive for safety, based on 3D printing

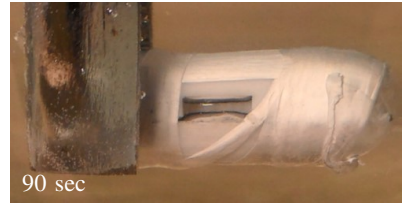

(a) Release of air from the capsule to let the liquid enter

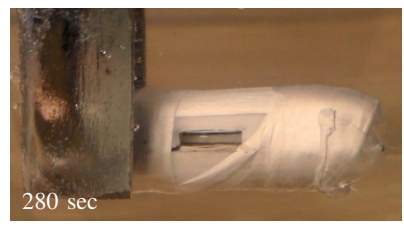

(c) End of liquid entrance in the capsule

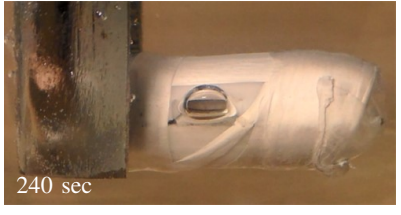

(b) Total release of the air from the capsule which results in the

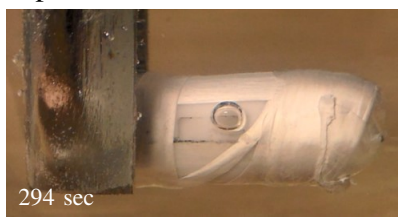

(d) Sealed capsule expulsion of an air bubble

Fig. 8: Decomposition of liquid entry into the capsule

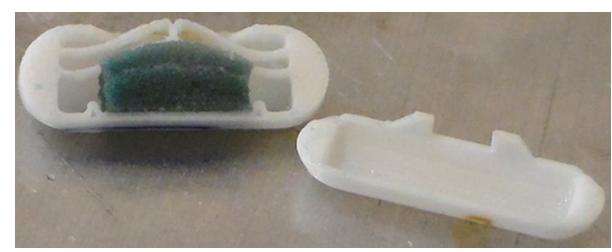

Fig. 9: Content of the capsule with swollen sponge after the experiment

for ease of integration, and uses PVA-based foam for both actuation and sampling. Initial evaluation in lab conditions is encouraging with first demonstration of sampling.

Future work will now be focused on the refinement of evaluation using liquids with viscosity close to the GI liquid. Evolution of the design will also be considered to maximize its robustness to manufacturing uncertainties. After integration of capsule coating, pre-clinical evaluation will then be considered.

\section{REFERENCES}

[1] Qin J. et al. A metagenome-wide association study of gut microbiota in type 2 diabetes Nature 490, 5560 (2012)

[2] Swidsinski A. et al. Mucosal flora in inflammatory bowel disease Gastroenterology 122, 4454 (2002).

[3] Ciuti G., Menciassi A., and Dario P.: Capsule Endoscopy: From Current Achievements to Open Challenges IEEE Reviews in Biomed. Eng., vol. 4 pp. 59-72, 2011.

[4] Yim S. and Sitti M.: Design and Analysis of a Magnetically Actuated and Compliant Capsule Endoscopic Robot, IEEE Int. Conf. on Robotics and Automation, pp. 4810-4815, 2011.

[5] Yim S., Gultepe E., Gracias D.H., and Sitti M.: Biopsy using a Magnetic Capsule Endoscope Carrying, Releasing, and Retrieving Untethered Microgrippers, IEEE Trans. on Biomed. Eng., vol. 61, pp. 513-521, 2014.

[6] Berger A., [online]. Tuit Nutrition, September 19, 2013. Available on: http://www.tuitnutrition.com/2013/ 09/Digestion-for-non-Dummies-Intro.html

[7] Thélu J., Cinquin Ph., Martin D., Soranzo T., Alcaraz J.P., Kweter J.M.: Device for intestinal fluid sampling. Patent $N^{\circ}$ FR1760069, 10/25/17, 2017.

[8] Cinquin P., Favier D., Alonso T., Bakri A., Khalef N., Thélu J. , Besson S., Martin D.: Dispositif de prise d'chantillon intestinal. Patent $N^{\circ}$ FR 1655187 filed by the UGA, 06/07/2016, 2016.

[9] Jin Qiu. (2003). An Electrothermally-Actuated Bistable MEMS Relay for Power Applications. PhD thesis. MIT. 